\title{
After Action Report: Idaho National Laboratory Annual Exercise June 10, 2015
}

July 2015

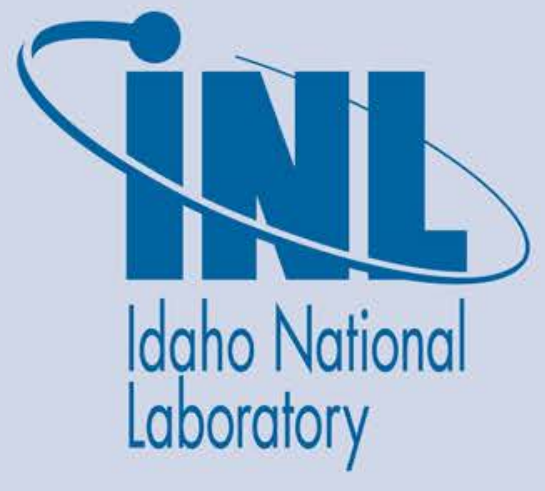

The INL is a U.S. Department of Energy National Laboratory operated by Battelle Energy Alliance 


\section{DISCLAIMER}

This information was prepared as an account of work sponsored by an agency of the U.S. Government. Neither the U.S. Government nor any agency thereof, nor any of their employees, makes any warranty, expressed or implied, or assumes any legal liability or responsibility for the accuracy, completeness, or usefulness, of any information, apparatus, product, or process disclosed, or represents that its use would not infringe privately owned rights. References herein to any specific commercial product, process, or service by trade name, trade mark, manufacturer, or otherwise, does not necessarily constitute or imply its endorsement, recommendation, or favoring by the U.S. Government or any agency thereof. The views and opinions of authors expressed herein do not necessarily state or reflect those of the U.S. Government or any agency thereof. 
INL/EXT-15-35780

Revision 0

\section{After Action Report: Idaho National Laboratory Annual Exercise June 10, 2015}

July 2015

Idaho National Laboratory Idaho Falls, Idaho 83415

http://www.inl.gov

Prepared for the

U.S. Department of Energy

Under DOE Idaho Operations Office

Contract DE-AC07-05ID14517 
This page intentionally left blank. 


\section{Emergency Management}

\section{After Action Report: Idaho National Laboratory Annual Exercise June 10, 2015}

INL/EXT-15-35780

Revision 0

July 2015

Submitted by Scott Barnes

Date

Exercise Director

Approved by Carl F. Farmer

Date

INL Emergency Management Manager 
This page intentionally left blank. 


\section{CONTENTS}

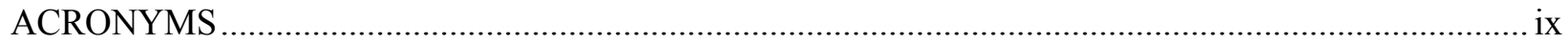

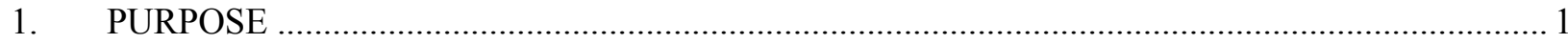

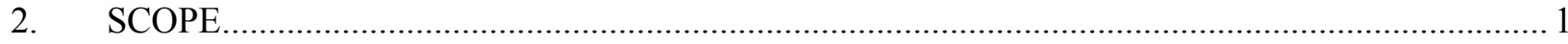

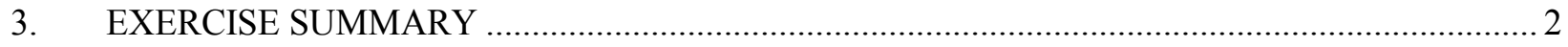

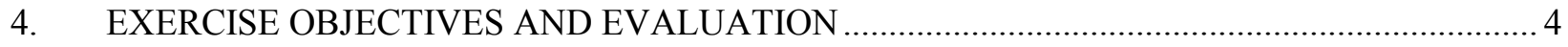

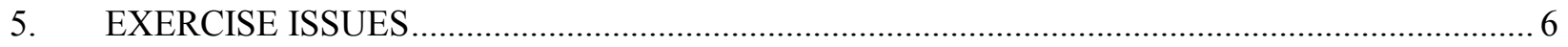

5.1 Emergency Response Organization Response ............................................................. 6

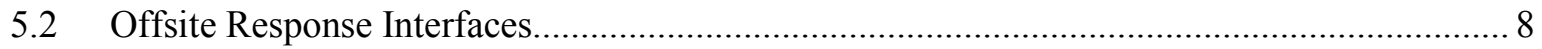

5.3 Emergency Event Categorization and Classification .................................................. 9

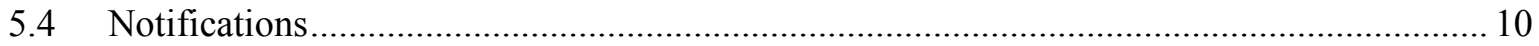

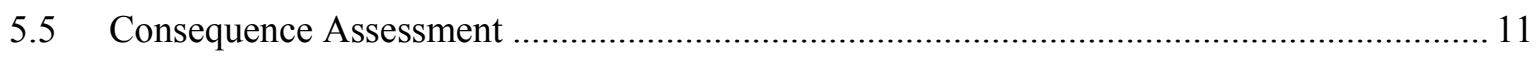

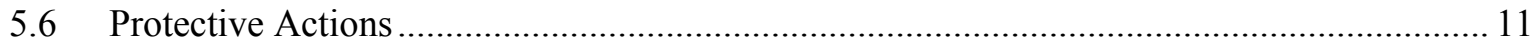

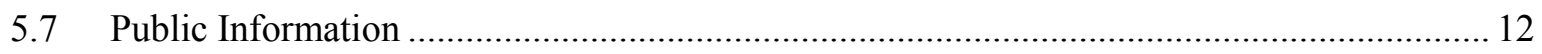

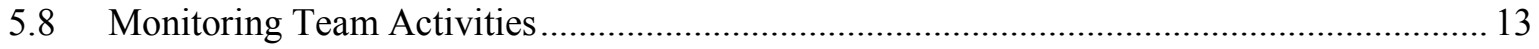

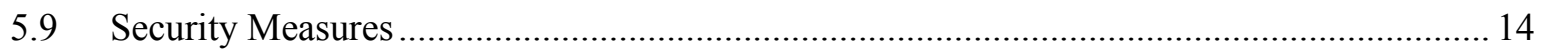

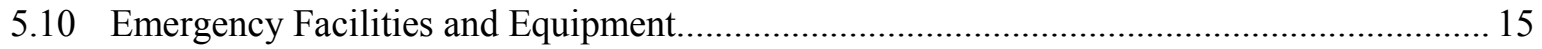

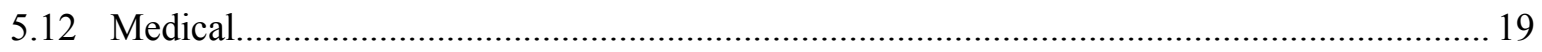

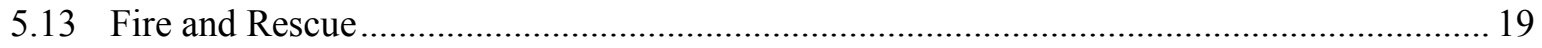

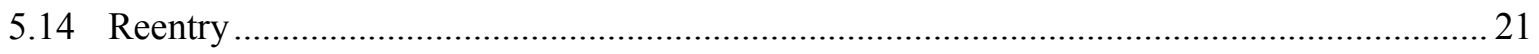

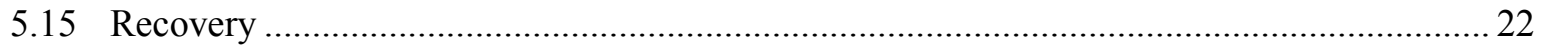

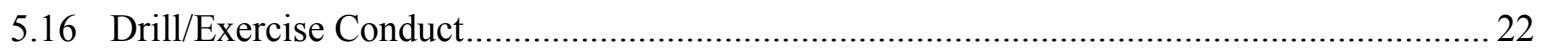

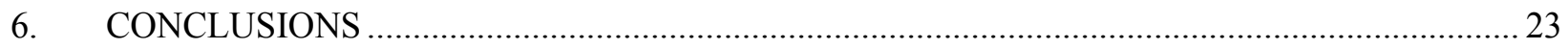

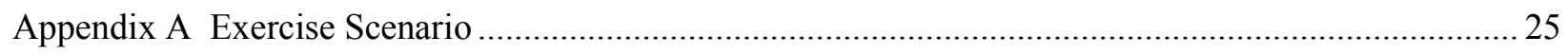

TABLES

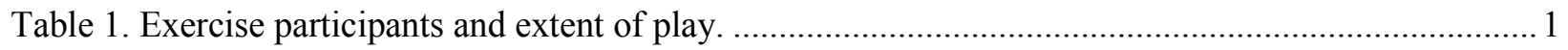

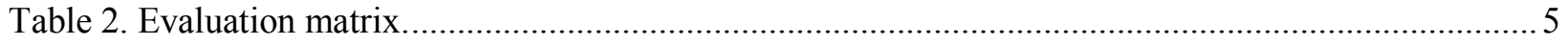


This page intentionally left blank. 


\section{ACRONYMS}

ATR

BEA

$\mathrm{BMH}$

CFA

CWI

DOE

DOE-HQ

DOE-ID

EAL

EAM

ECC

ED

EOC

ERO

EMS

FAC

FD

GPS

HPT

IAP

IC

ICP

INL

INTEC

ITG
Advance Test Reactor

Battelle Energy Alliance, LLC

Bingham Memorial Hospital

Central Facilities Area

CH2M-WG Idaho, LLC

Department of Energy

Department of Energy Headquarters

Department of Energy Idaho Operations Office

Emergency action level

Emergency action manager

Emergency Control Center

Emergency director

Emergency Operations Center

Emergency response organization

Emergency medical service

Fire Alarm Center

Fire Department

Global position system

Health physics technician

Incident Action Plan

Incident Commander

Incident command post

Idaho National Laboratory

Idaho Nuclear Technology and Engineering Center

Idaho Treatment Group, LLC 


\begin{tabular}{ll} 
JIC & Joint Information Center \\
NOAA & National Oceanic and Atmospheric Administration \\
NRF & Naval Reactors Facility \\
OE & Operational emergency \\
PA & Protective actions \\
PAR & Protective action recommendations \\
PF & Protective Force \\
PID & Public information director \\
PIDO & Public information duty officer \\
RadCon & Radiological control \\
RCT & Radiological control technician \\
SAE & Site area emergency \\
SMT & Site monitoring team \\
SMTC & Site monitoring team coordinator \\
SNF & Spent nuclear fuel \\
SPO & Security police officer \\
US & Wnited States \\
WCC & Warning Communications Center \\
\hline
\end{tabular}




\section{After Action Report: Idaho National Laboratory Annual Exercise June 10, 2015}

\section{PURPOSE}

On June 10, 2015, Idaho National Laboratory (INL), in coordination with the State of Idaho, local jurisdictions, Department of Energy Idaho Operations Office (DOE-ID), and DOE Headquarters (DOE-HQ), conducted the annual emergency exercise to demonstrate the ability to implement the requirements of DOE O 151.1C, "Comprehensive Emergency Management System." The INL contractor, Battelle Energy Alliance, LLC (BEA), in coordination with other INL contractors, conducted operations and demonstrated appropriate response measures to mitigate an event and protect the health and safety of personnel, the environment, and property. Offsite response organizations participated to demonstrate appropriate response measures.

Report data were collected from multiple sources, which included documentation generated during exercise response, player critiques conducted immediately after terminating the exercise, personnel observation sheets, and evaluation critiques.

Evaluation of this exercise served as a management assessment of the performance of the INL Emergency Management Program (IAS151187).

\section{SCOPE}

Exercise participants and their extent of play are shown in Table 1.

\begin{tabular}{|l|l|}
\hline \multicolumn{1}{|c|}{$\begin{array}{c}\text { Table 1. Exercise participants and extent of } \\
\text { play.Participants }\end{array}$} & \multicolumn{1}{|c|}{ Extent of Play } \\
\hline \multicolumn{2}{|c|}{ INL/BEA Organizations } \\
\hline $\begin{array}{l}\text { Advance Test Reactor (ATR) Complex } \\
\text { Emergency Control Center (ECC) }\end{array}$ & Limited participation: ECC control cell \\
\hline Bus Operations & Limited participation \\
\hline Central Facilities Area (CFA) ECC & Full participation \\
\hline INL Emergency Operations Center (EOC) & Full participation \\
\hline INL Fire Alarm Center (FAC) & Full participation \\
\hline INL Fire Department (FD) & Full participation \\
\hline Joint Information Center (JIC) & Full participation \\
\hline Occupational Medicine Directorate & Full participation \\
\hline INL Security & Full participation \\
\hline Warning Communications Center (WCC) & Full participation \\
\hline \multicolumn{2}{|c|}{ Other Site Contractors } \\
\hline $\begin{array}{l}\text { Idaho Nuclear Technology and Engineering Center } \\
\text { (INTEC) - CH2M-WG Idaho, LLC (CWI) }\end{array}$ & Limited participation: ECC control cell \\
\hline Idaho Treatment Group (ITG), LLC & Notifications only \\
\hline Naval Reactors Facility (NRF) - Bechtel Bettis & Notifications only \\
\hline
\end{tabular}




\begin{tabular}{|l|l|}
\hline \multicolumn{2}{|c|}{ Contiguous Counties } \\
\hline Bingham & Notifications only \\
\hline Bonneville & Notifications only \\
\hline Butte & Notifications only \\
\hline Clark & Notifications only \\
\hline Jefferson & Notifications only \\
\hline \multicolumn{2}{|c|}{ Federal Agencies } \\
\hline DOE-HQ Watch Office & Full participation \\
\hline DOE-ID & Full participation \\
\hline $\begin{array}{l}\text { National Oceanic and Atmospheric Administration } \\
\text { (NOAA) Air Resource Laboratory }\end{array}$ & Full participation \\
\hline $\begin{array}{l}\mid \\
\text { Idaho Bureau of Homeland Security }\end{array}$ \\
\hline Idaho State Communications Center of Idaho Agencies \\
\hline Idaho State Police & Notifications only \\
\hline INL Oversight Program & Notifications only \\
\hline \multicolumn{2}{|c|}{ Tribal Authority } \\
\hline Shoshone/Bannock Tribes & Notifications only \\
\hline \\
\hline Bingham Memorial Hospital & Full participation \\
\hline
\end{tabular}

\section{EXERCISE SUMMARY}

The exercise was conducted in two parts. Both Parts 1 and 2 were conducted on June 10, 2015.

The following initiating scenario background was presented to the actors at the scene in preparation for conducting Part 1. It was simulated that a spent nuclear fuel (SNF) shipment from the Advanced Test Reactor (ATR) Complex to the Idaho Nuclear Technology and Engineering Center (INTEC) was involved in a vehicle accident with a diesel fuel tanker. A semi-tractor with a flatbed trailer and a diesel tanker truck were staged on a road just north of the access road, where the event was simulated as occurring, to represent the accident scene. Actors portraying the injured were provided information about the accident, their injuries, and were moulaged in advance and staged where first responders would find them upon arrival. It was simulated that during the collision both drivers were injured and the SNF shipment trailer tipped on its side allowing the shipment cask lid bolts to shear and the lid to become dislodged. The tractor portion of the SNF shipment transport ignited on fire, which burned a security police officer (SPO) while checking on the driver. The SPO dropped to the ground and extinguished the flames on his clothing, receiving second-degree burns to approximately $20 \%$ of his body. The driver of the SNF transport vehicle, though injured during the accident, was able to get out of the cab of his truck through the passenger door. He and the injured SPO got a safe distance from the burning tractor. The SNF shipment and the diesel tanker were threatened by the fire, increasing the possibility of a radiation release. The driver of the tanker truck containing diesel fuel was still in the cab of his truck and was contaminated with radiological material. He was injured and responded only to verbal stimulus. By the time first responders arrived, the fire spread across the road and into the sagebrush. 
The drill started when INL Protective Force (PF) SPOs escorting the SNF transport saw the accident and reported it to Post 505. Simultaneously, the transportation job supervisor contacted the Warning Communication Center (WCC) and reported the accident. Both the WCC and Post 505 personnel contacted the INL Fire Alarm Center (FAC) who dispatched the INL Fire Department (FD). PF personnel responded, assisted injured and controlled access to the area. INL FD and emergency medical service (EMS) personnel arrived and established an incident command post (ICP), completed a scene size-up, and developed an incident action plan (IAP). Information on the event including shipment information was discussed with the SNF shipment job supervisor who remained nearby throughout the event to continue to provide needed information. A PF captain arrived on scene, established contact with the incident commander (IC), and coordinated security response efforts throughout the event.

The WCC contacted the emergency director (ED) and the support director and advised them of the situation. A determination was made to activate the Emergency Operations Center (EOC), Central Facilities Area (CFA) Emergency Control Center (ECC), and the Joint Information Center (JIC).

During this time, the fire continued to spread across the sagebrush. The SNF shipment and diesel tanker were being threatened by the fire increasing the possibility of a large release. Additional resources were requested by the IC, including Radiological control (RadCon) technicians (RCTs) from CFA, to monitor radiation levels at the scene, establish control boundaries, survey the injured along with emergency responders and their equipment on egress from the event area, and monitor for a release to the environment. Wildland units 2 and 3 were simulated as responding. INL FD personnel pulled fire hoses and established a fire attack posture in order to simulate putting out the burning tanker truck and protect the SNF shipment. As part of this demonstration, one wildland unit drove north of the event area simulating fighting the wildland fire and established a perimeter around the event area. The burning SNF tractor cab fire was put out and the wildland fire was eventually contained and then controlled.

The injured personnel, one of which was contaminated, were triaged, treated, and transported to CFA medical. The injured contaminated victim was transported offsite to Bingham Memorial Hospital (BMH). An INL RCT accompanied the contaminated victim in the ambulance to BMH. The INL Offsite Liaison met the ambulance at the hospital, as did a representative from the Idaho Department of Environmental Quality INL Oversight Program. The contaminated injured individual was successfully received by BMH staff. The INL RCT provided great direction and control during the transition, which helped BMH staff in their response actions. The remaining two injured personnel were transported to CFA medical for additional evaluation and treatment. They were then simulated as being transported to a local offsite hospital and treated as needed. Next-of-kin notifications were completed.

The CFA ECC, EOC, and JIC were staffed and declared operational in a timely manner. The CFA emergency action manager (EAM) declared an operational emergency using the applicable emergency action levels (EALs). Protective actions (PAs) were determined and simulated as implemented including the take shelter PA at the ATR Complex and INTEC. Accountability of field workers was conducted by CFA emergency response organization (ERO) personnel with a reminder for all field workers to stay clear of the area.

The CFA EAM provided event information during briefings conducted over the command bridge, which included the ED, ATR Complex ECC, and INTEC ECC control cell personnel. Categorization/classification, PAs/protective action recommendations (PARs), and notification functions were maintained by the CFA EAM throughout the exercise. Initial offsite notifications were completed by the WCC with the follow-up offsite notifications forms being completed but distribution to offsite agencies being simulated. 
Consequence assessment activities were conducted in the EOC planning room with results provided to the CFA EAM and the ED. As part of these activities, the INL site monitoring team (SMT) was successfully deployed to monitor for a release of radioactive material. During monitoring activities, it was determined no actual airborne release had occurred and the contamination on the injured truck driver came from some residual liquid spilled from the cask. The SMT provided information to the EOC assessment specialist(s) to verify no release had occurred, which helped them verify PAs were adequate. Environmental notifications were made.

Evacuations planning activities occurred and were coordinated with ATR Complex and INTEC ERO personnel. Members of the ATR Complex and INTEC control cells provided CFA ERO personnel applicable information that allowed them the opportunity to determine the number of evacuation buses needed, evacuation routes, and where to relocate the evacuees.

CFA ERO personnel initiated reentry planning activities in preparation of determining how to get the cask lid back in place. A good discussion occurred during this part of the exercise with first responders,

RadCon personnel, and applicable CFA ERO personnel. A determination was made that the objective of the first entry would be to obtain more information and pictures to assist with further reentry planning.

Media releases were developed by the EOC public information director (PID) and approved by personnel in the EOC. The media releases were then delivered to the JIC for simulation of release to the media. Actors portraying media interests, concerned members of the public, and family members continued to call JIC personnel for event information.

Once the fire was controlled, a successful turnover of IC responsibilities occurred between the battalion chief and the security PF captain. A briefing took place that included the RadCon supervisor, the SNF job supervisor, the battalion chief, and the PF captain. INL FD and EMS personnel then returned to station.

When a recovery manager was identified and the exercise director verified with the lead controllers that the identified objectives had been met and/or attempted; and when concurrence was obtained, part 1 of the exercise was terminated.

Hot washes were held at for each responding organization and ERO facility.

Part 2 involved transporting a contaminated injured person to BMH. While in route to BMH, INL EMS personnel contacted the BMH emergency room personnel and provided status information on the patient including contamination information. BMH personnel established contamination control tents in preparation to receive the patient. The transfer from INL EMS personnel to BMH personnel was completed with the assistance from INL RCT personnel.

The full scenario description is provided in Appendix A.

\section{EXERCISE OBJECTIVES AND EVALUATION}

During the exercise, 16 of the 16 standardized INL objectives were evaluated for INL using the appropriate demonstration criteria. Eight of the objectives were rated fully satisfactory, seven of the objectives were rated satisfactory with improvement needed, and one was rated as a weakness.

The ratings in Table 2 are based on the observations that follow in Section 0 . The gray shading within Table 2 represents an area not observed or rated. 
Table 2. Evaluation matrix.

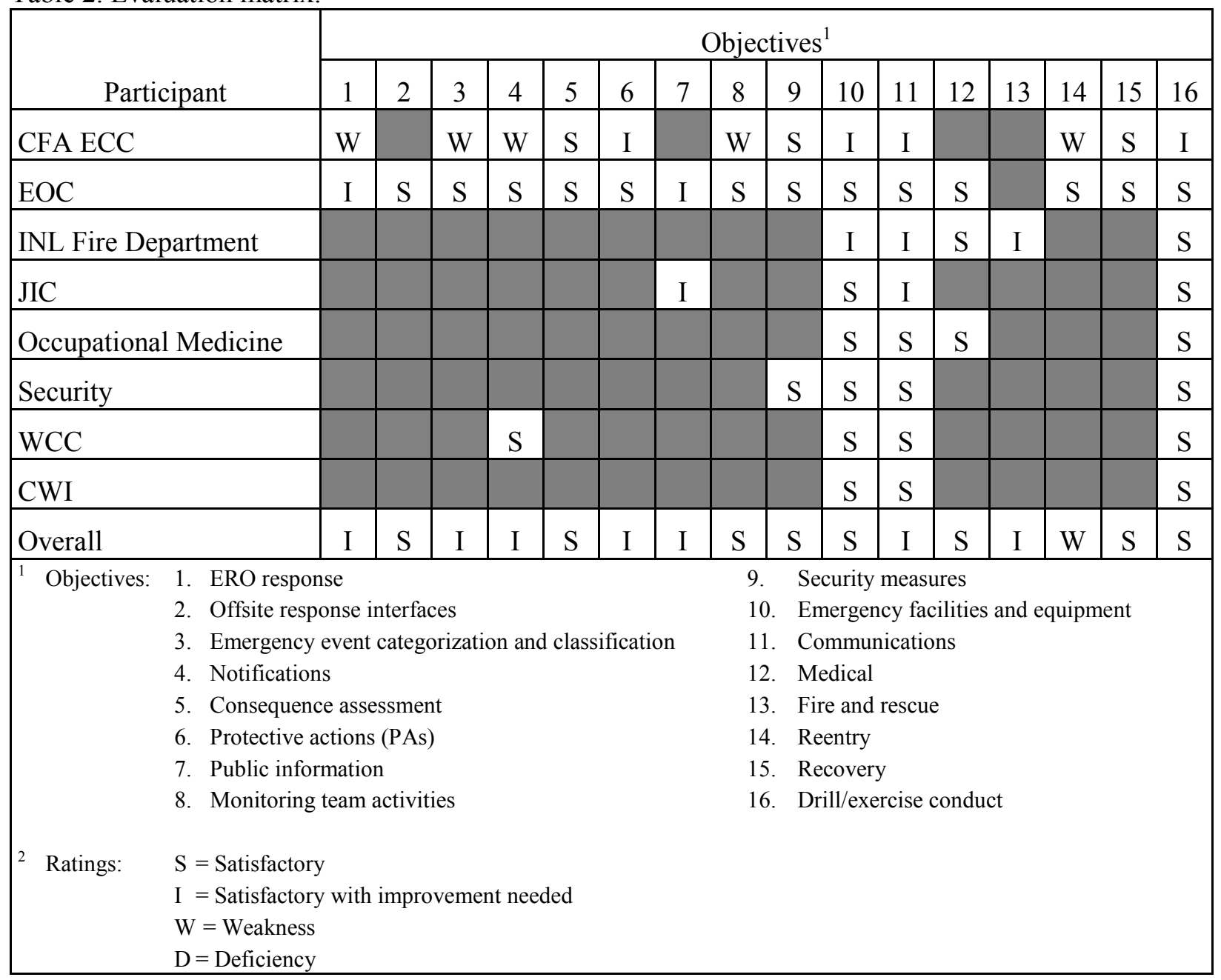




\section{EXERCISE ISSUES}

The following issues are specific to the evaluation of INL. Each item has been evaluated and entered into the INL issues management program currently referred to as LabWay.

\subsection{Emergency Response Organization Response}

Given the facility procedures/plan, the ERO will respond to, monitor, and evaluate the specific indicators of an emergency for mitigation of the consequences and bring the emergency situation under control.

\section{$\underline{\text { Discussion }}$}

The ED and support director received event information from the WCC and requested activation of the EOC, CFA ECC, and the JIC using applicable procedures and processes. The process for activation was followed and equipment functioned as designed. ERO personnel responded to their duty stations quickly, which enabled each to be declared operational in a timely manner. ERO response personnel utilized their applicable procedures, checklists, and logs to perform their functions.

Communication channels were established between the ERO response elements, which provided for transmittal of information between emergency facilities, but they were slow in getting communications established between the CFA ERO and IC. As information became available, briefings were provided to ERO personnel. Assignments and tasks were clear and made to the appropriate personnel. Overall, ERO staff successfully demonstrated their knowledge and use of response resources, capabilities, and how to access and use them. Support personnel were able to obtain and analyze the necessary information to support the ERO.

Designated personnel at the event scene and within the EOC successfully demonstrated direction and control of the response effort by establishing priorities and making task assignments while maintaining safety. Both the IC and the ED were able to demonstrate their knowledge of the resources available to them, which allowed for the mitigation of the event. Briefings were conducted with ERO personnel and the ED kept INL senior management apprised of the event.

\section{Issues}

\section{Weakness}

- Weakness 5.1.1: One CFA ECC non-decision maker ERO position was staffed by an unqualified employee. During the exercise, an ERO team member did not respond to the activation. The CFA support manager tried to get other qualified ERO personnel to staff the position. When he could not get a qualified person to help, he called an unqualified person to fill the planning communicator position. The planning communicator position is a non-decision maker and relays information passed across the phone line between the CFA ECC, the EOC, and any other ECCs that are activated.

Recommendation 5.1.1.1: Review the importance of staffing ERO positions with qualified ERO personnel during drills and exercises and how to determine which ERO personnel can be used to fill ERO positions that are not staffed, if one is not available. Laboratory Protection LabWay No. LP-CO-2015-1516 assigned. 


\section{Opportunities for Improvement}

- Opportunity for Improvement 5.1.1: During the initial activation, only the ED and support director were contacted by the WCC. An activation of the CFA ECC was requested during that conference call. During the decision making process of the conference call, communication were not established with the CFA EAM. If the CFA EAM had been included in the conference call, he would have had important event information that was necessary to help him in his decision making processes once he arrived at the CFA ECC.

Recommendation 5.1.1.1: Review the procedure and process for activating the ERO to determine if it should include having the support director and ED request the WCC to include the affected facility EAM in the conference call. Prior to closure of this action, provide the results to the Issues Screening Team (IST) to discuss updates to procedures as necessary and the need to provide lessons learned and necessary training to all EDs and support directors, if changes are made. Laboratory Protection LabWay No. LP-CO-2015-1517 assigned.

- Opportunity for improvement 5.1.2: During the event response, effective command and control was not demonstrated within the CFA ECC. Some ERO personnel, including the CFA EAM, were not very proactive in gathering event information nor did the CFA EAM provide direction to ERO personnel to use alternate methods to gather the information. When he was notified that there was a rad aspect to the event, he was slow in requesting additional resources for the ECC. It is recognized this lack of urgency could have been an issue of drillsmanship but the appearance was more a lack of familiarity with this type of an event. On a positive note, on his own initiative, the CFA planning manager, did contact the FAC to get some information and requested that FAC contact the IC and request that they contact the ECC.

Recommendation 5.1.2.1: Provide lessons learned training to include information regarding command and control activities and drillsmanship. Laboratory Protection LabWay No. LPCO-2015-1518 assigned.

- Opportunity for improvement 5.1.3: During the exercise, it was noted that CFA ECC ERO personnel, in general, did not follow their applicable position specific checklists, even when reminded to do so by the support manager. The support manager and the operations manager had their checklists out and were referring to them and following them. The information management coordinator had her checklist out and was using it, but most other team members in the CFA ECC followed them very little or not at all, relying on their memory as to what needed to be completed rather than utilizing the information on the checklist. In some cases, the checklist was completed after the exercise had been terminated, having the indications of being marked off as the team member would think "Oh, I did that" or "No, I didn't do that" rather than using the checklist during the exercise to remind them of what needed to be done. This practice could make observers wonder if the tasks being checked were completed or not during the exercise.

Recommendation 5.1.3.1: Provide lessons learned training to remind ERO employees of the importance of using the checklist throughout an event. Laboratory Protection LabWay No. LPCO-2015-1519 assigned. 


\subsection{Offsite Response Interfaces}

Given the facility procedures/plan, the ERO will coordinate and interface with response organizations to protect the environment and health and safety of the public.

\section{Discussion}

An effective interface was coordinated with Federal, state, tribal, and local agencies and organizations responsible for offsite emergency response was demonstrated. Initial event notifications were provided to local offsite agencies using established procedures and practices. Emergency facility equipment functioned to allow written and verbal communications among the various agencies. Follow-up notifications were simulated to local and offsite agencies.

The interface between INL EMS, INL RadCon, INL Offsite Liaison, INL Oversight, and BMH staff went very well. Great help and assistance was provided by INL personnel to the hospital staff. Feedback and/or suggestions provided to the hospital staff included having someone designated to take notes when deconning and collecting samples from the patient. This helped keep all of the samples straight. Other recommendation provided as feedback for hospital personnel was to practice donning and doffing decontamination clothing; utilize hot, warm, and cold zones; to make sure that the high-efficiency particulate air (HEPA) filter system is turned on during these types of events; and to use two meters to verify contamination and radiation levels on the victim and in the work areas for double verification, one used by INL personnel and one provided by INL Oversight. On a positive note, BMH trusted agents were able to keep maintain the secrecy of their participation in the drill as none of the other hospital staff responding to the exercise knew there was even going to be a drill.

Within the EOC, there was excellent interaction and communication between EOC ERO team members and INL Oversight personnel. There was a delay in the INL oversight personnel being notified of the event but this was a condition of drill participation since the Idaho State Communications Center was not participating beyond receiving offsite notification forms. In addition, for this exercise, communications were established between DOE-ID and DOE-HQ, as the watch office fully participated in this exercise. Good comments were received from DOE-HQ with a few minor comments such as their access to some WebEOC links. These activities are being worked through other processes and will not be addressed in this report.

For this scenario, effective interface and coordination with BMH, INL Oversight, and DOE HQ Watch Office were successfully demonstrated.

\section{Issues}

- No issues identified. 


\subsection{Emergency Event Categorization and Classification}

The ERO will accurately and promptly categorize and classify the operational emergency (OE) in accordance with the facility procedures/plan.

\section{Discussion}

Event categorization and classification was completed during the INL exercise but was slow in occurring. One of the causes was initial event information was not provided to the CFA EAM when the ECC activation occurred so he arrived at the ECC with no information as to why he was being activated. As the event continued to progress, event information continued to be slow coming into the CFA ECC. During this time, it appeared that the CFA EAM took more of a "let the information come to me" approach rather than being more proactive in gathering information. The CFA operations manager tried to contact the IC several times to no avail. He eventually contacted the FAC to get the initial event information. He also requested the FAC have the IC contact the CFA EAM. However, after those initial actions, he began to rely on what information came through the PF resources that were controlling access to the event area. This delay in communications caused a delay in getting the event categorized as an OE and/or further classified in a timely manner.

At approximately 32 minutes into the event, the CFA EAM had sufficient information to declare an unclassified OE using EAL TRN-ALL-1.OE.1, which description reads "hazardous material transportation-related fire outside an INL facility fence, and personnel injury or death is suspected or confirmed." With that information, an OE should have been declared within 30 minutes from the time of recognition. During this time, the EAM continued to try to determine if the radiation fields at the scene were $1 \mathrm{R} / \mathrm{hr}$ or $1 \mathrm{mR} / \mathrm{hr}$. There was also some confusion in determining which EAL to use, since CFA ERO team members were not familiar with some of the terminology used in the initiating event/condition description in the EALs such as "Advance Test Reactor (ATR) 30-W" vs. "Advance Test Reactor (ATR) 634-W". Personnel in the CFA ERO were not familiar with the term "Watts" and how they relate to reactor operations. At $\mathrm{T}+49$, the EAM chose an EAL that had been preidentified by the exercise director not to use because it would take the exercise away from the exercise scope. As a result, the exercise controller had to intercede and direct the EAM and his team to continue getting more information and reviewing the EALs. Approximately six minutes later, the EAM declared an alert, which by this time was 23 minutes after the time of event recognition with the time requirement for declaring a classified event being fifteen minutes instead of thirty thus making the declaration late. Even if the controller had not redirected the team, they would have been at the 17-minute mark to time of declaration.

Event conditions were monitored by ERO personnel and at $\mathrm{T}+86$, support personnel in the EOC recommended that the event be upgraded to a site area emergency (SAE) based on specific information about the contents of the SNF transport cask that was received. The event was upgraded to an SAE by the CFA EAM. The CFA EAM chose not to transfer the function of categorization/classification to the ED, which is allowed per procedure.

\section{Issues}

\section{Weakness}

- Weakness 5.3.1: Initial event categorization and/or classification did not occur in a timely and accurate manner.

Recommendation 5.3.1.1: Provide continuing/refresher training to the CFA ERO decision makers including the EAM, support manager, operations manager, and planning manager on determining categorization/classification. Consider using existing training course(s) such as EP350000 "PAR and EAL Review.” Laboratory Protection LabWay No. LP-CO-2015-1520 assigned. 
Recommendation 5.3.1.2: Review the EAL initiating event/condition description applicable to ATR SNF shipments to determine if they should be written in a more descriptive format or with an explanation of what "Watts" means. Laboratory Protection LabWay No. LP-CO-2015-1521 assigned.

\subsection{Notifications}

Given the facility procedures/plan, the ERO will report emergencies and conduct follow-up notifications to the appropriate organizations within the required time.

\section{$\underline{\text { Discussion }}$}

Initial offsite notifications were completed at 0954, which is 14 minutes after the event was initially classified as an alert, just making the 15-minute time requirement. When the event was upgraded to a SAE, offsite notifications were completed according to procedure. Follow-up offsite notifications were completed approximately every hour thereafter until the exercise was terminated. A termination notification was not completed.

There were errors made on the follow-up notification forms some of which were corrected and some were not. The errors that were corrected were done following applicable procedures and process. The errors that were not corrected included the sequential numbering of the forms and some of the information required in block 6 dealing with the classification level, time of declaration, and the EAL(s) used. Usually the initial form is considered the first or \#1, with the follow-ups being number sequentially after as they are submitted. In this case, the person completing the forms numbered the first "follow-up" as \#1 instead of \#2. The other error on the form had to do with the time of declaration on the follow-up forms and the level of classification. First, it appears that the time documented in block 6 of the form was the time the form was completed instead of the time of declaration as it changed each time a subsequent form was completed. The second error in block 6 was the box that was marked each time was the block for an "Alert" while the EAL used was for a SAE, which was the classification declared.

On a positive note, the individual completing the notification form, though new in the position, was very diligent in ensuring that the time requirements for completing notifications was met. The PAs that were listed for the SAE were the ones implemented. The CFA EAM retained the notification function throughout this exercise and did not transfer these responsibilities to the ED. Though in most instances it is preferred to transfer the notification function to the ED, it is not a requirement.

Next-of-kin notifications were simulated for this exercise. The medical director in the EOC went through the process to determine if the injured victims were BEA employees. Once the INL EOC medical director received victim information and identified them as BEA employees, the rest of the next-of-kin process was simulated.

\section{Issues}

\section{$\underline{\text { Weakness }}$}

- Weakness 5.4.1: During the annual ERO exercise, there were multiple errors on the emergency notification form that were not corrected or caught during the review process.

Recommendation 5.4.1.1: Provide training to the CFA EAMs and CFA support managers on completing and reviewing notification forms. Laboratory Protection LabWay No. LP-CO2015--1522 assigned.

Recommendation 5.4.1.2: Review notification forms to determine if the numbering of the forms can be engineered out to prevent confusion on the required numbering scheme. Laboratory

Protection LabWay No. LP-CO-2015- 1523 assigned. 


\subsection{Consequence Assessment}

Given the facility procedures/plan, the ERO will assess actual and potential onsite and offsite consequences of an emergency.

\section{$\underline{\text { Discussion }}$}

Initial and on-going consequence assessment was successfully demonstrated by using default assessment data and then continuing to monitor the event and update as new information was received.

Default information was used in conjunction with current meteorological information to verify initial PA distances were adequate. Since there was not an airborne release for this event, EOC assessment specialists were proactive in identifying additional hazards that might be involved that could be released to the environment and what the PAs should be by acquiring and reviewing applicable information on the ATR canal water that was in the cask to determine if airborne could be an issue from the water. EALs were verified using event information provided from CFA ERO personnel and personnel on scene using the planning bridge and consequence assessment form. As additional event information was received, consequence assessment personnel continued to monitor the event and provide recommendations to ERO personnel.

Meteorological information was available, utilized throughout the exercise, and used in the consequence assessment process including weather forecast capabilities. During this exercise, there was a lot of field data received making the consequence assessment process go smoother.

Overall, this objective was successfully demonstrated with the performance of the consequence assessment process and updates provided to the appropriate decision-makers.

\section{Issues}

- No issues identified.

\subsection{Protective Actions}

Given the facility procedures/plan, the ERO will respond to emergency conditions to protect onsite personnel and the public by implementing specific, predetermined actions.

\section{Discussion}

At the initiation of the exercise, the job transport supervisor followed procedures, contacted the WCC, the ATR, and the INTEC shift supervisor/EAM, and explained what had happened. Both facilities were simulated as being placed in a take shelter. WCC personnel completed their event notification process to activate the ERO. When the IC arrived, the job transport supervisor reported what actions had been taken. Responders were told that their electronic dosimetry was alarming. They initiated response actions to retrieve the first two of three injured persons. They were slow in getting to the third injured individual as a result of the fire.

ERO personnel arrived at the EOC and CFA ECC and determined and implemented PAs for the event. Initially, it was determined that a 100 meter evacuation distance was required based on the criteria for an alert. As more information was received, the event was upgraded to a SAE and the evacuation distance was changed to 478 meters per the information contained in the EALs. This distance does not encompass either ATR Complex or INTEC, and the EALs do not provide any other PA direction beyond those distances. As a result, the INTEC EAM was not provided additional information on PAs until the first command bridge briefing approximately 50 minutes into the event. 
RadCon support was requested by the INL FD to assist at the scene. When the RCTs arrived, they reported to the IC, began monitoring activities, and implemented PA distances from the event area. They conducted surveys and provided decontamination assistance for response personnel and their equipment. This was a positive activity since CFA ECC does not have a monitoring team assigned to provide this type of field or "facility" monitoring as in the fenced in facilities located on the INL. CFA RadCon support had not been involved in any training or drills with the ERO. This was known prior to the development of the drill. However, it was determined by the drill director to conduct the exercise with current conditions and provide opportunities for improvement identified during the exercise. Even with not having participated in a drill or exercise, CFA RadCon personnel responded and effectively assisted in event control and mitigation.

Accountability at the scene was completed quickly, but field worker accountability took longer than expected. Part of the reason was the evacuation distance was only 478 meters and PAs were implemented and accountability completed within the PA area. It was the additional field worker notification directing persons to stay clear of the event area that did not occur until about the 70-minute mark.

PAs and PARs were not transferred to the ED and remained with the CFA EAM for the duration of the exercise. This is allowed but not the preferred approach during events.

\section{Issues}

\section{Opportunities for Improvement}

- Opportunity for Improvement 5.6.1: During the annual exercise, event information regarding protective action should have been provided to potentially impacted facilities in a timelier manner.

Recommendation 5.6.1.1: Provide lessons learned training to reinforce the importance of providing event information emphasizing PAs and PARs to other facilities that might be impacted by the event. Laboratory Protection LabWay No. LP-CO-2015-1524 assigned.

\subsection{Public Information}

Given the facility procedures/plan, the ERO will demonstrate an emergency public information program.

\section{$\underline{\text { Discussion }}$}

The JIC was activated at approximately 0910 and declared operational at 0922, taking about 12 minutes for them to assemble and be ready to operate.

Three news releases were developed, approved, and distributed, while a fourth news release was developed and approved during the duration of part 1 of the exercise. This is exceptional in terms of information sharing. Typically, the EOC only releases two to three news releases.

The headline for news release \#2 conflicted with the content of the release: one injured versus two (in body of news release). This could have been edited in the JIC with concurrence of PID. The JIC did not ask to edit the headline.

The Public Information Duty Officer (PIDO) from DOE-ID was not available in the EOC until 1007. News release \#1 is preapproved and so that did not impact the first news release being sent to distribution, which was simulated. However, when news release $\# 2$ had been routed to all other required reviewers and approved, no PIDO was available for final review and approval. At 0952, the controller allowed the PIDO approval to be simulated with other DOE officials' concurrence and was allowed to be distributed by the JIC for simulated distribution. Another PIDO was eventually identified and responded to the EOC.

No mock news conference was conducted in conjunction with this exercise. 
Issues

Weakness

- Weakness 5.7.1: The assigned PIDO from DOE-ID did not respond to the activation notification.

Recommendation 5.7.1.1: Ensure the PIDOs are aware of the importance of their participation in an exercise. This position plays an important role in public information distribution and emergency response. DOE-ID to reconcile.

\section{Opportunities for Improvement}

- Opportunity for Improvement 5.7.1: There was conflicting information between the headline and the body of a news release during the annual exercise.

Recommendation 5.7.1.1: Remind JIC personnel and PIDs that information can be edited by the JIC with the concurrence of the PID. Laboratory Protection LabWay No. LP-CO-2015-1525 assigned.

- Opportunity for Improvement 5.7.2: The PID was persistent in trying to gather event information when there was no new information to be provided by the ED or the support director.

Recommendation 5.7.2.1: Remind PIDs it is acceptable to be persistent in gathering event information, but remind them when the ED and/or support director indicate they do not have any more current information, to be patient and allow ERO personnel to have more time to monitor the event and be briefed as event conditions change or more information becomes available, as opposed to continually asking after they are told there is not any more information. Laboratory Protection LabWay No. LP-CO-2015-1526 assigned.

\subsection{Monitoring Team Activities}

Given the facility procedures/plan, the ERO provided facility/site monitoring teams in support of consequence assessment activities.

\section{$\underline{\text { Discussion }}$}

The SMT was activated and arrived at the CFA ECC in a timely manner. They conducted equipment checks per procedure and made contact with the site monitoring team coordinator (SMTC) in the EOC. The SMT was deployed based on coordinates provided by the SMTC in coordination with the consequence assessment specialists.

The SMT utilized applicable personal protective equipment and effectively deployed to the given areas and conducted sampling and monitoring activities. Several sample/data points were provided to the assessment specialist to assist them in verifying adequate PAs had been implemented.

During this exercise, two items for improvement were identified. One found early was the units of measure for global position system (GPS) locations used by various responding organizations were different. Some were using one latitude/longitude system such as the degree/decimal/minutes system while others were using degrees/minutes/seconds for determining location. This caused some confusion and could have potentially led to the deployment of the SMT into a plume or a high rad area. Another is that CFA does not have RadCon personnel assigned as a response element of the ERO. 
INL fenced facilities have RadCon personnel assigned either as part of the ERO either on a facility monitoring team (FMT) or as a technical specialist function that is called in for events involving rad. During this exercise, CFA RadCon personnel were requested by the IC and they responded to the scene. Neither the CFA RCTs nor their supervisors have been included in ERO response activities such as drills and exercises in the past. It is noted that the SMT is used for plume monitoring and verification not on scene response activities. CFA RCTs effectively monitored the event, established boundaries, and an egress area. They effectively monitored emergency response personnel and their equipment at the established egress point. Decontamination activities were simulated.

Issues

\section{Opportunities for Improvement}

- Opportunity for Improvement 5.8.1: GPS location units used by emergency response organizations are different. Some are using one of the latitude/longitude systems such as the degree/decimal minutes system while others were using degrees/minutes/seconds for determining location. Others might be using a different system such as the Universal Transverse Mercator (UTM) coordinate system.

Recommendation 5.8.1.1: Determine what GPS location units are being used by INL emergency response organizations and national resources such as Radiological Assistance Program teams. Determine which should be used by INL emergency response organizations and make the necessary changes to procedures, equipment, and software as needed. Laboratory Protection LabWay No. LP-CO-2015-1527 assigned.

- Opportunity for Improvement 5.8.2: A FMT does not exist to respond to rad events within the purview of CFA ERO response areas and responsibilities.

Recommendation 5.8.2.1: Determine the level of need for a FMT for CFA ECC response actions to include, if needed, a FMT, a facility monitoring team coordinator, necessary equipment, procedure changes, training, and drills. Laboratory Protection LabWay No. LP-CO-2015-1528 assigned.

\subsection{Security Measures}

Per facility procedures/plan, security will respond to, monitor, and evaluate the specific indicators of an emergency for mitigation of the consequences and to bring the emergency situation under control.

\section{$\underline{\text { Discussion }}$}

INL Security personnel escorting the transport followed applicable procedures to contact emergency responders, which mobilized appropriate security response personnel. The security PF shift captain arrived on scene and established a unified command with the IC. They quickly established access control to the affected areas and strictly controlled access to event area throughout the exercise.

Security consulted appropriate emergency response resources for initial event information. As additional information was received, additional resources were deployed to control access around the event area and protect personnel. Security personnel utilized resources to assess the hazards to their responders and established safety protocols to keep their responders out of the rad areas. 
An effective communications network was utilized between security responders and the IC to ensure the safety of personnel and the protection of assets involved in the event. This included working with RCTs and their supervision at the scene to determine safe standoff distance for long-term monitoring of the event area. Good discussion occurred between EMS personnel and security personnel on what to do with the SPO's personal equipment when they are injured and need to be transported.

Security personnel assigned in the CFA ECC and the EOC effectively demonstrated their response actions including establishing communications network with each other and the shift captain co-located with the IC.

\section{$\underline{\text { Issues }}$}

- No issues identified.

\subsection{Emergency Facilities and Equipment}

Demonstrate the adequacy of facilities, equipment, displays, and other materials to support emergency operations in accordance with the facility procedures/plan.

\section{$\underline{\text { Discussion }}$}

Facilities and equipment were used effectively during the exercise to support emergency response operations. Equipment used in the INL WCC to facilitate response activities, including the use of the Everbridge System to activate the INL ERO elements, functioned properly. Written documentation was available and utilized throughout the exercise. Facilities and equipment were available and are adequate to support emergency response activities. Responders were able to find the supporting references and materials to support their activities. Sufficient forms and other supplies were available.

CFA ECC, INL EOC, and WCC personnel used WebEOC to document and track significant events. Procedures and position-specific checklists were on-hand, up-to-date, and available for use by INL ERO members. iMaps, which is a Google Maps based system used during drills, exercises, and actual events, to depict facility and building layouts, was used in the CFA ECC and the EOC to orient ERO personnel with the layout of the event area and location of response elements. This system is still in the testing phase so it was evident that personnel are still becoming familiar with the smartboards and the use of the iMaps system displayed on them. Vehicles were available for use to evacuate nonessential personnel at the ATR Complex and INTEC, if needed.

The SMT arrived at the CFA ECC, performed equipment checks, and found no issues. During their monitoring activities in the field, all equipment functioned as designed. It is noted in addition to section 5.8, "Monitoring Team Activities," of this report of the potential discrepancy in the units of measure used by emergency response organizational when using latitude and longitude coordinates to determine locations of personnel and/or equipment. Corrective actions are addressed in section 5.8.

As documented in section 5.8, CFA does not have an ERO element to respond to rad events like this transportation accident as do other INL fenced facilities. CFA RCTs and their supervision responded and conducted appropriate activities, but had to rely heavily on their supervisor's direction and did not have the type of equipment readily available for this type response. A comprehensive list of equipment has been identified since this exercise was conducted and is being incorporated into the corrective action listed under this section.

SMT and CFA RCTs had problems with cell phone signal strength making it hard to maintain communications during the exercise. Several calls were dropped or hard to hear. 
All equipment in the INL EOC Planning Room was operational. Web-based maps, WebEOC, and weather software were used in the response actions.

Overall, INL FD equipment and facilities were adequate. INL FD command officers utilized Department of Transportation Emergency Response Guidebook for initial PAs and then collaborated with RadCon for radiological exclusion areas. Firefighters utilized DMC 2000 personal electronic dosimetry during response actions. All were knowledgeable on their use and alarm set points.

INL JIC equipment was fully functioning throughout the exercise. Resources were easily accessible and JIC personnel utilized them as appropriate. Facilities and equipment were adequate for response in the INL EOC Public Information area.

Overall, the equipment in the INL WCC, INL EOC, and CFA ECC worked as designed and provided for a coordinated response to the event. All of the facilities' infrastructure equipment, such as lighting and ventilation, functioned properly and adequately supported the response activities. This objective was successfully demonstrated with weaknesses and opportunities for improvement noted below.

\section{$\underline{\text { Issues }}$}

\section{Weakness}

- Weakness 5.10.1: CFA RCTs did not have the equipment readily available to allow them to respond to rad events in a timely manner.

Recommendation 5.10.1.1: Identify the needed equipment for RCT response, determine funding options, and acquire the equipment. Laboratory Protection LabWay No. LP-CO-2015-1529 assigned.

Recommendation 5.10.1.2: Identify a storage area for RCT response equipment. Laboratory Protection LabWay No. LP-CO-2015-1530 assigned.

- Weakness 5.10.2: Cell phone calls between the SMT and the SMTC were dropped or hard to hear. The RadCon supervisor also had the same problem while on scene trying to direct RadCon response actions. This appeared to be due to weak signal strength that could present problems during an actual response requiring multiple users to use cell phones.

Recommendation 5.10.2.1: Verify and identify signal strength capability implemented on the INL. Laboratory Protection LabWay No. LP-CO-2015-1531 assigned.

Recommendation 5.10.2.3: Submit LabWay for tracking and trending. Laboratory Protection LabWay No. LP-CO-2015-1532 assigned.

\section{Opportunities for Improvement}

- Opportunity for Improvement 5.10.1: SMT has a difficult time using radios and cell phone while wearing full-face respirators.

Recommendation 5.10.1.1: Evaluate and provide recommendations to EM manager on alternative types of equipment that can be used with the radios and or cell phones while wearing respirators. Laboratory Protection LabWay No. LP-CO-2015-1533 assigned.

- Opportunity for Improvement 5.10.2: Some INL FD personnel seemed hesitant or unfamiliar with the use of their rad monitoring equipment. 
Recommendation 5.10.2.1: Review the use of rad monitoring equipment with INL FD responders. Laboratory Protection LabWay No. LP-CO-2015-1535 assigned.

\subsection{Communications}

Given an $\mathrm{OE}$, communications capabilities are managed in support of emergency operations to ensure prompt and appropriate flow of accurate information in accordance with facility procedures/plan.

\section{$\underline{\text { Discussion }}$}

Communications among responders in the field were effective with incident command providing direction to the responders in an effective manner. Repeat backs or three-way communications were used during the exercise in most areas with improvement needed in the CFA ECC. This includes using position titles when talking to each other in the ECC or EOC. As noted in previous sections, the CFA EAM did not receive event information until after activation and arriving at the CFA ECC. Even then, communications were slow in being established between the CFA ECC and the IC. The CFA operations manager made several attempts to establish communications with the INL FD on scene and was unsuccessful; this delayed getting the CFA EAM event information. The CFA operations manager eventually contacted the FAC to get the information. While the operations manager was attempting to establish communications with the IC, the security leader was able to get information from the security captain on scene and relay it to the CFA EAM and CFA operations manager. Overall, it appeared that some personnel in the CFA ECC lacked a sense of urgency in obtaining necessary information to clear up some of the confusion caused by unknown or conflicting information. This would have helped them in their decision-making processes. It was noted in the hot wash that an on scene communicator other than INL FD responders might be needed to allow for better communications between on scene and the CFA ECC. Other facilities at the INL use this method.

Communications between the ECCs and the EOC were established but CFA ECC was slow in getting someone to staff the planning communicator position. While waiting for personnel to arrive at the CFA ECC, the support manager would get on the conference call and try to provide information but this was not very effective. This slowed down the communications between CFA and the other EROs. Eventually a person was located to staff the position, which made the communications between facilities and the EOC effective. Communications within the EOC was effectively demonstrated. The ED used repeat backs extensively during command bridge briefings to verify information.

INL EMS personnel established communications with CFA medical and effectively communicated needed patient information. Effective communications were established between INL EMS and the local hospital, $\mathrm{BMH}$, during transport of one of the patients to $\mathrm{BMH}$. Once the ambulance arrived at $\mathrm{BMH}$, excellent communications were established and demonstrated between BMH staff, INL RCTs, and EMS personnel. The INL FD, CFA Medical Clinic, WCC, and EOC medical director used the appropriate communication paths and protocols to relay victim information for next-of-kin notifications.

When INL Security and INL FD personnel established unified command, they shared information that enabled INL EMS personnel to effectively mitigate the event and control access into and around the area. When CFA RadCon personnel arrived, a good briefing occurred and information was shared that enabled the RCTs and their supervision to perform their functions effectively.

The WCC and INL FAC emergency reporting equipment worked as designed. WCC and FAC personnel effectively handled calls and alarms per applicable procedures and protocols.

The PID assistant consistently communicated with JIC personnel and the INL PID. Relevant information was shared between the INL EOC and JIC. JIC team members are trained and prepared to use emergency communications protocols during drills, exercises, and actual events. 
Communication systems in the EOC Public Information room worked to provide prompt and reliable communications inside the EOC and with the JIC. All of the equipment, including speakers to hear briefings, and all other systems worked well. Responders communicated well with the JIC on the telephone.

\section{Issues}

\section{Weakness}

- Weakness 5.11.1: CFA ECC personnel did not effectively use three-way communications including identifying themselves and others by position title when sharing information within the CFA ECC.

Recommendation 5.11.1.1: Provide opportunities for CFA ERO personnel to practice three-way communications protocols during training sessions or conduct drills that emphasize effective communications practices. Laboratory Protection LabWay No. LP-CO-2015-1536 assigned.

- Weakness 5.11.2: CFA personnel were slow in establishing communication channels between the CFA ECC and IC.

Recommendation 5.11.2.1: Provide lessons learned training emphasizing the importance of quickly establishing communications channels with the IC and what alternate routes can be used if the initial attempts fail. Laboratory Protection LabWay No. LP-CO-2015-1537 assigned.

Recommendation 5.11.2.2: Evaluate the need of having a designated on scene communicator position associated with the CFA ERO that will respond to the event scene and establish communications with the CFA ECC and implement, if deemed necessary. Laboratory Protection LabWay No. LP-CO-2015-1538 assigned.

\section{Opportunities for Improvement}

- Opportunity for Improvement 5.11.1: INL FD and security personnel need to establish better communication processes and/or protocols. It appeared that the FAC was informing the battalion chiefs when the SPO or the ECC was attempting to make contact. This can be a command and control issue or a training issue.

Recommendation 5.11.1.1: Work with INL FD and Security to determine the appropriate use of talk groups and if additional action is needed. Present recommendation to Issues Screen Team prior to closure. Laboratory Protection LabWay No. LP-CO-2015-1539 assigned.

- Opportunity for Improvement 5.11.2: Facility factsheets in JIC are not current or were not located.

Recommendation 5.11.2.1: Replace factsheets in JIC with updated/current factsheets. Ensure that JIC staff knows where to locate current factsheets - hardcopy in the JIC and electronically on the external web. Links can be shared with the media and public. Laboratory Protection LabWay No. CO 2015-1540 assigned. 


\subsection{Medical}

Given the facility procedures/plan, the medical response personnel will respond to, monitor, and evaluate the specific indicators of an emergency for mitigation of the consequences and bring the emergency situation under control.

\section{$\underline{\text { Discussion }}$}

EMS personnel carried out their response activities in a safe manner. The initial event information dispatched them to what they thought was a transportation accident with possible injuries. While en route, they were notified that a fire had ignited and that there were injuries. By the time the FD responders had arrived on scene, the SNF transport vehicle was fully involved. Diesel from the transport vehicle fuel tank had shot across the road and ignited the sagebrush on fire on the opposite side of the road.

INL FD and EMS personnel sized up the scene and established an ICP. The first two injured personnel were retrieved from the event area. Once the victims were rescued and moved to an ambulance, the care was exemplary. However, there was a delay in the rescue of the third victim. The fire across the road initially cut off the injured diesel fuel shipment tanker truck driver from EMS personnel. As noted in section 5.13, this impacted effective response actions by EMS and INL FD personnel as it appeared that the IC began to prioritize fire attack over rescue of the patient. This was not the prioritization of contamination over life but lack of assigning resources in a manner consistent with life priority.

The ambulance crews provided transportation of the injured personnel to the CFA medical facility and one of the offsite hospitals, BMH. During transports, ambulance crew personnel maintained effective communication with personnel at the receiving facilities, while providing pertinent information such as vital signs and information on the individual's physical condition including an assessment regarding internal or external contamination. Receiving facilities were kept apprised of the estimated time of arrival. Upon arrival at both CFA Medical and BMH, EMS crews provided appropriate patient reports and transferred care to the receiving facility. Transition of care went smoothly.

EMS personnel followed proper communication channels to provide CFA medical personnel with applicable information, who in turn provided the medical director the information on patient status, which enabled him to complete next-of-kin notifications.

CFA Medical's identification of the severity of injuries was accurate and timely. They identified the patient's needed surgical consultation and rapid transport to a trauma center. They performed a brief examination to ensure no injuries or conditions were present that needed to be managed prior to transport, then ensured a timely and appropriate transport decision.

Overall, this objective was successfully demonstrated.

\section{Issues}

- No issues identified. 


\subsection{Fire and Rescue}

Per procedures/plan, the fire and rescue responders will respond to an event involving fire or hazardous material, mitigate the consequences, and bring the situation under control.

\section{Discussion}

The initial event information dispatched INL FD personnel to the scene. INL FD and EMS personnel arrived and established incident command, began scene size up, and incident action plan development. The first two accident victims were evaluated and moved to a safe location for treatment. For the third victim, response was slow as wildland fire fighting activities were engaged prior to the victim being checked, as the wildland unit drove right past him without even checking to see if he was injured. It was noted during the exercise that a few INL FD personnel lacked a sense of urgency in making their initial response. This may have been a result of them thinking it is only an exercise, making their initial response actions more methodical and less urgent. Response crews were effective in addressing life safety hazards and established tactics to reduce risk to the responders and public.

The IC worked with INL Security personnel to aid in isolating the emergency scene and controlling access by placing physical barriers to control traffic around the event area. Though it did not play a factor in the mitigation of the event it would have been more effective if physical barriers would have been set up to separate the different zones. For example, hot, warm, and cold zones for rad control purposes. Instead, only one physical barrier was established and that was at the egress point where responders were monitored for contamination. This condition was a result of CFA not having a designated monitoring team assigned to respond directly to the event. A backup engine company from INL FD Station 1 arrived on scene to assist with response activities.

Effective communications between responders were used throughout the exercise. Response teams effectively kept the IC briefed on event conditions and patient status. Incident command was established and effective throughout the event. The INL FD battalion chief established incident command on arrival at the scene. One item that could have been demonstrated more effectively would have been in organizing the command structure this may have made the patient rescue activities occur in a timelier manner instead of focusing on fire attack. The battalion chief also kept moving around the scene area instead of staying in one place making it more difficult to locate him when face-to-face information needed to be shared.

Overall, this objective was successfully demonstrated with opportunities for improvement identified below.

Issues

\section{Opportunities for Improvement}

- Opportunity for Improvement 5.13.1: INL FD personnel lacked a sense of urgency during their initial response.

Recommendation 5.13.1.1: Remind INL FD drill/exercise participants during safety briefings to participate as if the event is real. Laboratory Protection LabWay No. LP-CO-2015-1541 assigned.

- Opportunity for Improvement 5.13.2: The IC did not do a good job at organizing the command structure, thus patient rescue was delayed while fire attack was being initiated. Additionally, the IC should have stayed in one place, rather than moving around the scene.

Recommendation 5.13.2.1: Remind appropriate INL FD personnel of the IC structure. Laboratory Protection LabWay No. LP-CO-2015-1542 assigned.

- Opportunity for Improvement 5.13.3: Players did not exemplify the importance of playing in drills and exercises as if it were a real event. 
Recommendation 5.13.3.1: Provide lessons learned training on the importance of playing in drills and exercises as if it is a real event. Laboratory Protection LabWay No. CO 2015-1543 assigned.

\subsection{Reentry}

The ERO will demonstrate development of a reentry plan in accordance with the facility procedures/plan.

\section{Discussion}

The need for a reentry plan was discussed between the CFA EAM and ERO members. It soon became evident that the CFA ERO has not had much experience doing reentry planning. Most of their response experience has been dealing with wildland fires where reentry planning is non-existent. The EAM announced readiness to commence reentry planning but still was not sure of his radiological status. He expressed a desire to have the ATR EAM plan reentry and then have CFA implement the plan since ATR had more radiological engineering and skilled personnel resources with experience in loading casks. He called the ATR EAM and proposed the idea to him. He got off the phone saying that the ATR EAM would call the CFA planning manager to discuss resources needs and support.

With reentry planning languishing, the ECC controller prompted the CFA EAM to bring people into the ECC from the incident scene to assist with reentry planning. The controller then coached the ERO on who to bring in. (At this point, the reentry phase looked very much like a training event, instead of an evaluated exercise to determine readiness.)

The CFA planning manager did step up to the plate and direct the reentry planning effort by obtaining the applicable procedure and emergency work permit. He then coordinated reentry planning with the applicable players and completed page 1 of Form 150.05, "INL Emergency Work Permit." Since an actual reentry was not planned, page 2 was not completed, which provides the framework for conducting a pre-job brief.

This event provided a great opportunity for CFA ERO to work out ownership of lead responsibilities in reentry and recovery and identify the needed support from other facilities. Since this was an INL event rather than the smaller scale fenced facility type events, it enabled ERO team members to think big picture with CFA as the lead with support on the technical side from ATR and EOC ERO personnel. It also provided for an exercise in logistics to recover from this type of an event.

After the initial problems getting recovery planning started, the CFA ERO was able to complete the reentry planning for an initial reentry that would have allowed them to obtain vital information for additional reentries to secure the SNF, get the lid secured and the cask and trailer upright.

\section{$\underline{\text { Issues }}$}

\section{Weakness}

- Weakness 5.14.1: CFA ECC personnel were not familiar with reentry planning.

Recommendation 5.14.1.1: Provide opportunities for CFA ERO personnel to practice reentry planning through a variety of scenarios that will allow them to plan reentry activities from simple to complex tasks. Laboratory Protection LabWay No. LP-CO-2015-1544 assigned. 


\subsection{Recovery}

Given the facility procedures/plan, the ERO will demonstrate part 1 of the recovery planning process for an emergency at the affected facility by identifying a recovery manager.

\section{Discussion}

At 1210 , the CFA EAM, in conjunction with facility management, identified a recovery manager. Though this was late in the exercise and had to be prompted by the ED during a command bridge briefing, it is acceptable to have happened during that time. The CFA ERO had just completed the initial reentry planning meeting and were getting their arms around the big picture of the event when the command bridge occurred. During the briefing of the event, the ED asked the CFA EAM if a recovery manager had been identified, which reminded the CFA EAM that one needed to be identified.

Overall, this objective was successfully demonstrated.

\section{Issues}

- No issues identified.

\subsection{Drill/Exercise Conduct}

Write, conduct, and evaluate a drill/exercise that will emphasize facility-specific emergency events and response activities and minimize the use of generic, nonspecific simulations in accordance with the facility procedures/plan.

\section{Discussion}

A technically accurate emergency event scenario with clear measurable objectives was presented and responded to by facility ERO teams and response organizations. The scenario emphasized a challenging series of emergency events with provisions for realistic free-play during response actions. Event data and information presented during the exercise provided the sequence of the events in a realistic manner, and was representative of actual facility conditions. Appropriate vehicles were used as props that provided good visual/practical aids to make the scenario more realistic. All three victims were moulaged prior to the initiation of the exercise to emphasize their injuries and provided instructions on how to act when the emergency responders arrived. During the exercise development, it was realized that the CFA ERO does not have the same support from RadCon as the INL facilities located on the INL within the confines of a facility fence. These facilities, as applicable, have a FMT with a facility monitoring team coordinator located in the ECC as part of the ERO to direct monitoring activities within their facility boundary. CFA ERO does not have this capability. They have to rely on any RCTs that happen to be in the area and respond when requested. A decision was made to test the response capability as it currently stood rather than have a contingency developed. During the conduct of the drill, opportunities for improvement were identified and are documented in the applicable sections.

Controllers were utilized to facilitate performance of the players and ensure safe and effective conduct of the exercise. Cue cards were well-written, providing applicable relevant information on the initiating event and follow-up event to keep the exercise moving in the right direction and at the correct times, and kept to a minimum. In the CFA ECC, there were several situations where a controller coached the team members when they were slow or struggling where if they were allowed to continue their actions could have got the exercise off track. In the opinion of one observer, the controller let the situation go as long as he could before intervening. In the EOC, there were instances of controllers and exercise observers talking which was distracting to exercise participants. 
Exercise controllers and evaluators were briefed and instructed on their assignments prior to the exercise. Exercise conduct evidenced much preparation and training. Controllers and evaluators were jointly involved in the pre-exercise briefings. There were a sufficient number of controllers and evaluators to provide coverage for all participating areas.

Evaluators were utilized to observe, evaluate, and critique performance of the exercise. Immediately following termination of the exercise, "hot wash" critiques were conducted with all exercise participants at each of the evaluated areas. An evaluator/controller critique was also conducted to discuss exercise performance.

\section{Issues}

\section{Opportunities for Improvement}

- Opportunity for Improvement 5.16.1: There were numerous examples of coaching or prompting by the CFA ECC controller during the exercise and too much talking or interaction between controllers and observers in the EOC. It appeared this was done to keep the drill advancing such as when the ERO struggled when reentry planning was initiated.

Recommendation 5.16.1.1: Evaluate and recommend alternate methods for using the facility planner as a controller in an area other than their facility ECC or allowing them to participate as a player during some evaluated drills and/or exercises. Laboratory Protection LabWay No. LPCO-2015-1545 assigned.

- Opportunity for Improvement 5.16.2: A couple of instances occurred where exercise participants and actors could have played their roles more realistically. For example, some INL FD personnel lacked a sense of urgency during their initial response. They appeared to have an attitude of "Oh, another drill." In another example, one of the SPOs who was on scene was a little on the sarcastic side in his verbal responses. During one activity, he was standing on the running boards of one of the semi-tractors involved in the accident watching an injured victim when a wildland unit drove within approximately 25 feet of him and he said nothing or did nothing to get their attention to respond to his area to help the victim. When asked by the controller why, he responded in essence, "They can come to me; I'm not going to chase them." When asked if that is what he would do in a real situation, he responded, "No, I would yell at them to come." The controller asked why they did not do that for the drill so they can respond appropriately? To which he got their attention and they responded.

Recommendation 5.16.2.1: Tracking and Trending. Laboratory Protection LabWay No. LPCO-2015-1546 assigned.

\section{CONCLUSIONS}

Overall, a successful evaluated exercise was conducted at INL. Several good things were demonstrated such as the triage, treatment, and transporting of the victims. Particularly in the demonstration of our ability to transport and hand-off of the contaminated injured patient to BMH. The INL RCT, who escorted the patient to BMH, was very effective in assisting with the decontamination efforts at BMH. The INL offsite liaison officer responded to $\mathrm{BMH}$, as did INL Oversight personnel where they worked hand in hand with BMH staff. Good communications were demonstrated within the EOC between ERO team members and with DOE-ID and the INL Oversight representative. Additionally, we successfully demonstrated our ability to effectively establish communication with DOE-HQ and share information using WebEOC. Offsite notifications were completed in a timely manner and in accordance with applicable procedures. 
The INL FD and EMS personnel responded to the event area, established incident command and successfully triaged, treated, and transported the three injured victims. As resources became available, they were able to control the fire. IC requested RadCon assistance and they responded to the event area, established a control area, and effectively performed monitoring and decontamination activities.

PF personnel responded, established access control to and around the event area, and worked effectively with the IC. After the INL FD response was over, a transfer of responsibilities of IC occurred between the INL FD battalion chief and the PF captain. The PF captain, acting now as the IC, worked with RadCon personnel to ensure the SPOs were safe in their locations as they established a perimeter around the cask and fuel.

ERO teams were activated in accordance with procedures. The event was declared an operational emergency by the CFA EAM and PAs were determined and implemented. Communications were established between the applicable ERO members and outside agencies, between the ECCs and the EOC, and between the CFA ECC and IC.

Several opportunities for improvement and some weaknesses have been identified during the course of developing and conducting this exercise. These include the need for a more organized RadCon response effort. Though the RCTs and RCT supervisor who responded did an excellent job, they do not have ready access to equipment for these types of response or communications lines back to the ECC. They were briefed by the IC when they arrived on scene but they should be briefed before they are deployed so they have a better understanding of what they are responding to and what the hazards are. Another area where improvement is needed is in the lines of communications between the IC and the CFA ECC. They were slow to materialize and caused delays in receiving needed information to categorize and/or classify the event. This could have caused delays in determining and implementing PAs. Lastly, the CFA EAM did not effectively demonstrate command and control actions during the exercise. In some cases, he appeared to make decisions by majority consent. Though these items for improvement may look significant, other ERO personnel, using applicable procedures and processes, stepped up and were proactive in getting information and implementing PAs without waiting for a declaration of an OE or recommendations from the CFA EAM, as they normally would in an event of this nature.

This exercise provided a great avenue to practice interactive response capability where one facility, CFA, had to rely on another facility for resources and material at risk information as demonstrated by their willingness to involve ATR Complex personnel who are subject matter experts. CFA ERO personnel also had to rely on EOC assets to help them in determining the correct EALs, as the CFA ERO did not have expertise in SNF shipments and how the applicable EALS related to the different types of SNF shipments that occur across the INL. Most events on the INL, including those within facility fences are event specific and there are sufficient facility experienced personnel to respond. This particular event involved material from one facility being transferred to another facility. Once the material leaves a fenced facility boundary, it becomes CFA ERO responsibility for emergency event response because it is traversing CFA's area of responsibility. This unfamiliarity caused some delays in determining what was in the cask so they could match it with the EALs and declare the event as an OE. As a result, the SNF shipment EALs will be reviewed to see if they can be written differently to make OE determination more efficient and effective.

Overall, even with the uncertainties and unknowns that were experienced during this exercise, the INL ERO and emergency responders successfully demonstrated their ability to respond to and mitigate emergency events on the INL. 


\section{Appendix A}

Exercise Scenario 
This page intentionally left blank. 


\section{Appendix A}

\section{Exercise Scenario}

\section{Background}

INL is a multi-program laboratory owned by the United States (U.S.) government and managed and operated by BEA for DOE-ID. The primary mission of INL is to ensure the nation's energy security with safe, competitive and sustainable energy systems and unique national and homeland security capabilities. INL works with national and international governments, universities, and industry partners to discover new science and develop technologies underpinning the nation's nuclear and renewable energy, national security, and environmental missions. INL includes BEA-managed and -operated facilities that are located on the Site. The Site is an 888-square-mile area of open, sagebrush-covered desert land in southeastern Idaho, approximately 25 miles west of Idaho Falls. Facilities managed and operated by other contractors for DOE are also located on the Site.

CFA is located on the Site, approximately 2 miles from the intersection of U.S. Highways 20 and 26; 49 miles west of Idaho Falls; and 22 miles east of Arco, Idaho. CFA is the main service and support center for other primary INL facility programs. Major activities at CFA consist of INL site-wide programmatic support such as transportation, maintenance and crafts, capital construction, environmental and radiological monitoring, security, fire protection, warehousing, and calibration laboratories. Other INL facilities located outside of CFA that are considered part of CFA for emergency response purposes include Critical Infrastructure Test Range Complex and Experimental Breeder Reactor-I. CFA is also responsible for emergencies involving transportation accidents, wildland fires, and any other emergency events within the Site boundary, except the area contained inside of designated Site facility boundaries, including state and federal highways intersecting the Site boundary.

\section{$\underline{\text { Start }}$}

Following is simulated as happening: A SNF shipment from ATR Complex to INTEC is involved in a vehicle accident with a diesel fuel tanker. During the collision, the shipment trailer tips on its side, allowing the shipment cask lid bolts to shear and the lid to become dislodged. The tractor portion of the shipment transport ignites on fire and threatens the contents of the tanker and the SNF cargo. The driver of the tanker truck, who is still in the cab of the truck, is contaminated, injured, and non-responsive. The driver of the SNF transport vehicle is injured, as is one of the INL protective force (PF) personnel. The fire begins spreading across the sagebrush. The SNF shipment is threatened by the fire, which increases the possibility of a radiation release.

\section{$\underline{\text { Part } 1}$}

PF personnel with the transport contact Post 505 and report the accident. Simultaneously the transportation job supervisor contacts the WCC. Both the WCC and Post 505 personnel contact the INL FAC, which dispatches the INL FD. PF personnel control access to the area. INL FD and emergency medical personnel arrive, complete a scene size up, and develop an action plan.

The WCC contacts the emergency director (ED), the support director, and the CFA Emergency Action Manager (EAM) and advises them of the situation. A determination is made to activate the EOC, CFA ECC, and the JIC. 
The injured personnel, one of which is contaminated, are triaged, treated, and transported to CFA medical. One contaminated victim will be transported to a local hospital, if participating. The fire continues to spread across the sagebrush. The SNF shipment is still threatened by the fire with the probability of a release increasing.

Additional resources are requested by the incident commander (IC) including RCT or health physics technicians (HPT) to monitor for radiation release to the environment. Wildland units 2 and 3 are simulated as responding. Engine Company's 1 and 4 (engine 4 could be simulated because of operational needs) continue to mitigate the burning tanker truck and cool the SNF shipment.

CFA ECC is declared operational as is the EOC and JIC. The CFA EAM declares an operational emergency using the applicable Emergency Action Levels (EALs). PAs are determined and implemented. ATR Complex ECC and INTEC ECC monitor the event. Offsite notifications are completed and the CFA EAM provides a briefing over the command bridge.

Injured personnel are transported to CFA medical and Bingham Memorial Hospital and treated as needed. Notification of next-of-kin will be completed.

Accountability of field workers is completed.

PF personnel working with the IC continue to control access to the event area. The INL SMT is deployed to monitor for a release of radioactive material. Other organizations will participate as needed for monitoring purposes.

During monitoring activities, it is determined a release has occurred. Information will be provided to the EOC assessment specialist(s) to verify PAs are adequate using applicable plume modeling software. Environmental notification will be made. Planning activities occur on how to get the cask lid back in place and contain the potential runoff from the fire mitigation activities that are headed to the Big Lost River.

Command Bridge briefings are held as needed during the event.

Categorization/classification, PAs/PARs, and notification functions may be transferred from the CFA EAM to the ED.

Press releases are developed by the EOC public information director and approved.

If an evacuation of ATR Complex or INTEC is requested, it will be simulated as will the deployment of evacuation buses. Facility ERO personnel will provide applicable information to CFA ERO personnel to allow them the opportunity to determine the number of evacuation buses needed and where to relocate the evacuees.

The support director requests the WCC make radio announcements about the status of the event periodically.

Consequence assessment activities are conducted in the EOC planning room with results provided to the CFA EAM and the ED.

The WCC receives emergency notification forms throughout the event. Initial notifications to offsite agencies will be completed, but transmittal of follow-up notification forms to offsite agencies is simulated. 
Once the fire is out, the wildland fire contained, and the SNF shipment cooled, reentry planning will occur to verify the contents of the SNF shipment are secure.

When a recovery manager is identified and has an outline of a recovery plan started, event termination discussed and concurrence from the ED and DOE-ID is requested and given, the exercise director will verify with the lead controllers that the identified objectives have been met and/or attempted; and when concurrence is made, part 1 of the exercise will be terminated.

The WCC receives a termination notification form, transmits it to participating agencies, and makes applicable announcements to close the drill window.

Hot wash critiques are conducted.

\section{$\underline{\text { Part } 2}$}

If participating, the local area hospital will receive the contaminated injured person at their facility, complete the decontamination process, and begin assessment activities. Applicable INL resources will be available to assist as needed in the monitoring and decontamination of the victim. 